\title{
Uno de los desafíos de la educación superior; competencias ciudadanas para el postconflicto en Colombia, marco legal
}

Daniela Alejandra Alba Useche ${ }^{2}$

Luis Alejandro Padilla Beltrán ${ }^{3}$

Recibido: 05-07-2016

Aceptado: 07-09-2016

\section{RESUMEN}

Al hablar de formación ciudadana en Colombia, se habla de la Constitución Política de Colombia de 1991, que constituyó a Colombia en una "democracia participativa"; a partir de ella, se ha generado una serie de leyes que buscan una verdadera formación ciudadana como lo pide ésta, teniendo como objetivo formar un ciudadano demócrata y participativo; es por eso, que la importancia de la formación en competencias ciudadanas en los estudiantes se vuelve un imperativo constitucional y legal para poder tener una democracia constituida por ciudadanos; con esto busca superar las secuelas de las constantes guerras civiles del siglo XVIII, el conflicto armado empoderado desde el siglo XIX, sus resurgimientos periódicos a lo largo del XX y su existencia en lo que va corrido del XXI; con lo que se espera que la finalización de los diálogos de paz del gobierno con la guerrilla de las FARC- EP y con el ELN; permitan hablar de un escenario de postconflicto o pos acuerdo.

Por tal razón hay que resaltar que, desde la promulgación de la Constitución Política de Colombia de 1991, se han implementado leyes como la Ley 30 de 1992, Ley 107 de 1994, Ley 115 de 1995, así hasta llegar a la Ley 1732 de 2014 de la creación de la "Catedra para la paz"; que obligan a que las competencias ciudadanas sean enseñadas en las aulas de clase, tanto en la educación básica y media, como en todas las

1. Artículo resultado del Proyecto de Investigación DIS-1508 "Formación ciudadana en instituciones de Educación Superior; en paz y para la paz; dentro del contexto de la política pública "Estudio de caso UMNG", financiado por la vicerrectoría de investigaciones de la Universidad Militar Nueva Granada..

2. Profesional en Relaciones Internacionales y Estudios Políticos, Univer $\neg$ sidad Militar Nueva Granada. Joven investigadora grupo PIREO, Programa de Relaciones Internacionales y Estudios Políticos a Distancia.

Correo:

3. Magister en Educación, Abogado. Docente tiempo completo Programa de Relaciones Internacionales y Estudios Políticos a Distancia.

Correo: luis.padilla@unimilitar.edu.co 
Instituciones de Educación Superior. Pero a la hora de ponerlas en práctica en la sociedad del pos conflicto, los objetivos deben estar encaminados a poder realizar aportes como ciudadanos en la vida democrática, teniendo en cuenta cumplir lo pactado en dichos diálogos y la futura consolidación y sostenimiento de la paz en el tiempo, fundamentado en el respeto de la vida y del que piensa diferente.

Palabras clave: Ciudadanía, Competencias Ciudadanas, Educación Superior, Postconflicto.

\section{One of the challenges for higher education; Civic competences for post- conflict in Colombia, legal framework}

\section{ABSTRACT}

Speaking of training citizens in Colombia, refers to the Constitution politics in Colombia in 1991, which constituted to Colombia in a "participatory democracy"; from it, it has generated a series of laws seeking a true citizen training and ask this, aiming to form a national democratic and participatory; that is why the importance of skills training citizens in the students becomes a constitutional and legal imperative in order to have a democracy made up of citizens, this seeks to overcome the consequences of the constant civil wars of the 18th century, empowered from the 19th century armed conflict, their periodic comebacks throughout the 2oth and how are kept so far run 21st; but expected and hopes come to an end by the present Government peace dialogues with the guerrillas of the FARC - EP and the ELN; then you can speak of a scenario of post-conflict or pos agreement.

For this reason must be emphasized that, since the promulgation of the Constitution, Colombia policy, 1991, laws such as the law 30 of 1992, 107 law of 1994, law 115 of 1995, have been implemented as well until you reach the law 1732 of 2014 from the creation of the "Department for peace"; that they have forced to the citizenship skills are taught in classrooms both in basic education and media in all the Institutions of higher education. But when it comes to put them into practice in society 
in a possible conflict pos, the objectives must be aimed to be able to make contributions as citizens in democratic life, taking into account comply with what has been agreed in these dialogues and future consolidation and sustainability of peacekeeping at the time, based on respect for life and who think differently.

Keywords: Citizenship, civic skills, higher education, Post-Conflict.

"Prefiero el título de Ciudadano al de Libertador, porque éste emana de la guerra aquel emana de las leyes"

Simón Bolívar

\section{Introducción}

El tema de la educación superior en Colombia, requiere un análisis en su proceso evolutivo dentro de un país abatido por las guerras civiles del siglo XVIII y el conflicto armado que de manera continua se encuentra vigente desde mediados del siglo XIX hasta nuestros días; Y como las Instituciones de Educación Superior (IES) con sus egresados deben impactar en la transformación social de manera real y conocer su transformación en la situación democrática colombiana; dentro del ámbito académico, conocer el proceso de formación de los estudiantes en cuanto a las competencias ciudadanas y cómo sería el funcionamiento y alcance de estas dentro de un escenario de postconflicto o pos acuerdo; ya que esta herramienta fomenta "programas orientados a la formación de colombianos en el respeto a los derechos humanos, a la paz y a la democracia" (Ministerio de Educación, 2012), por lo que se debe tener presente a toda la comunidad académica, para poder actuar de acuerdo a una misma visión hacia la calidad en educación y en formación de sus estudiantes de manera integral.

Por medio del presente artículo, se busca realizar un análisis del marco legal de la formación ciudadana en la educación superior en Colombia, para entender cuáles son los aportes que se proporcionarían al poner en 
práctica las competencias ciudadanas en un país ad portas de firmar el acuerdo para la terminación del conflicto armado que vive Colombia; para de ésta generar un nuevo escenario social, político, cultural, entre otros, en el país y causar de esta manera un impacto positivo en la sociedad y el desarrollo de la democracia participativa tal y como lo pide la Constitución Política de Colombia, con el fin de que la paz sea realmente sustentable y sostenible en mediano y largo tiempo.

\section{Método}

El presente artículo de reflexión, es resultado de un estudio que surge con el proyecto de investigación "Formación ciudadana en Instituciones de Educación Superior; en paz y para la paz; dentro del contexto de la política pública" Estudio de Caso UMNG", elaborado por el grupo de investigación PIREO de la Universidad Militar Nueva Granada, el cual busca analizar el nivel de competencias ciudadanas en los estudiantes de primero y último semestre de la misma, mediante un enfoque cualitativo y descriptivo no experimental que permitió realizar un análisis de los resultados y de esta manera poder crear una propuesta para preparar de una mejor manera a los estudiantes en su paso por las diferentes Instituciones de Educación Superior en cuanto al tema de competencias ciudadanas. Se generó una revisión bibliográfica de algo más de cincuenta documentos nacionales e internacionales obte $\neg$ nidos a través de bases de datos virtuales como ProᄀQuest, Dialnet, Jstor, páginas oficiales de entidades gubernamentales y no gubernamentales y libros; los cuales se seleccionaron los más pertinentes para elaborar la fundamentación teórica de la temática: "competencias ciudadanas y educación superior en el postconflicto".

\section{Educación en Colombia}

Para el estudio se tomaron los inicios de la educación en Colombia en el siglo XIX, donde en la mayoría del territorio colombiano la vida se desenvolvía en el área rural, por lo que la educación mayormente iniciaba en los hogares, con escasos medios y la mayor parte del tiempo se empleaba en labores propias del campo; por tal razón principalmente el conocimiento que adquirían los niños y jóvenes era en dichas labores; 
"en 1940 Colombia era todavía un país agrario, con más del 70\% de su población situada en el sector rural" (Cajiao, 2004, p. 32). Y la poca educación superior se centraba en las principales ciudades.

Es por eso que en Colombia a nivel pedagógico, la enseñanza política se inició en la casa siguiendo una tradición familiar, y luego esta se dio en la escuela primaria para los que podían ingresar a la educación básica ya que aún no se tenía la suficiente cobertura, dado que "el nivel de escolarización era todavía muy precario y la permanencia de niños y niñas en el sistema se reduce a dos o tres años, sobre todo en el sector rural" (Cajiao, 2004, p. 32).

En 1927 se dictó una ley donde se consagra la educación como un derecho; los niños tenían la opción de recibir la enseñanza desde la casa o desde una escuela, siempre y cuando fuera gratuita como estipula el artículo 4 de la ley 56 de 1927:

Artículo $4^{\circ}$ Los padres, guardadores y demás personas que hagan las veces de los padres están obligados a proporcionar a los niños un mínimo de educación que comprenda las bases necesarias para la vida en materia de instrucción intelectual, moral y religiosa, cívica y física tal como las fijará el Decreto reglamentario de esta Ley, pero quedan en libertad de escoger los medios de dar cumplimiento a esta obligación en alguna de las formas siguientes: a) Haciendo instruir a los niños en una escuela, ya sea pública o privada, y b) Procurándoles enseñanza en el hogar.

Es así como los niños en las áreas rurales podían adquirir nuevos conocimientos y habilidades enfocadas a las labores domésticas y al campo, mientras se desarrollaban dentro de una población que empezaba a crecer hacia las áreas urbanas, con el agravante de una población amenazada por la violencia en el sector rural. No se conoce con claridad el inicio y formación de este grupo guerrillero, pero si es claro que su origen se da en medio de las luchas agrarias, en la década de 1920, causada por los terratenientes en varias regiones del país y con el paso del tiempo, con la prohibición de cultivar ciertos productos, afectando a los campesinos y al país en general (Vega Cantor, 2004, p. 28).

Debido a las continuas luchas agrarias que no pudo resolver la Ley 200 de 19364; posteriormente aparece el grupo guerrillero Fuerzas Armadas 
Revolucionarias de Colombia (FARC), inicialmente como defensor de los afectados de esa violencia en contra del desplazamiento y despojo de sus tierras, producto de las guerras bipartidistas.

La violencia bipartidista que ha llevado a Colombia en un desgaste social, político y económico, es de las cosas más insólitas del mundo, "EI episodio de La Violencia es el otro origen de las FARC. (...) Suscitada por la disputa por el poder a la que se entregan los dos partidos tradicionales, el liberal y el conservador" (Pécaut, 2009, p. 37).

Adicionalmente, por el acelerado crecimiento de la población, el gobierno se ve en la obligación de crear más escuelas, tanto en los pueblos como en las ciudades y como la educación debía promoverse como un derecho, es entonces, cuando al Estado le toca preocuparse por el tema. "en Colombia, (...) la educación se convierte en una prioridad, no sólo por su papel en el desarrollo, sino como forma de consolidar el sistema democrático" (Cajiao, 2004, p. 36).

Hasta cuando llega la Constitución de 1991 que considera en el artículo 67 que:

La educación es un derecho de la persona y un servicio público que tiene una función social; con ella se busca el acceso al conocimiento, a la ciencia, a la técnica y a los demás bienes y valores de la cultura.

En cuanto a la educación básica y media regida por la Ley 115 de 1995; se da una cobertura mayor a la de la Educación Superior. Se realiza mediante instituciones de educación tanto pública como privada. Adicionalmente por un lado, el Estado ofrece una educación superior a través de la oferta con sus universidades públicas y por la otra con subsidio a la demanda con el programa "ser pilo paga"; donde los estudiantes eligen en que universidad quieren ingresar sin importan que ésta sea pública o privada; sin embargo no alcanza a cubrir toda la demanda ya que este programa no alcanza a cubrir el tres por ciento de la demanda (3.\%); esto a su vez indica que no hay una cobertura total ni universal en la educación superior; por ello debe ser una prioridad para el Estado ampliar esta cobertura a todos los colombianos y que puedan acceder al derecho a la educación, como lo dicta el artículo anteriormente mencionado de la Constitución Política de Colombia.

una reforma agraria, para frenar la violencia bipartidista por ese tema, después de lo que se conoce como la Republica Conservadora que se extiende desde 1886 hasta 1930, año en que los conservadores pierden la hegemonía, y se obtiene el primer gobierno de corte liberal desde 1886. 
Con la Ley 30 de 1992, se organiza la educación superior en Colombia, y es la ley a la que deben someterse todas las instituciones de educación superior tanto públicas como privadas; y orienta lo que espera el Estado a través de ellas que el estudiante sea educado tal y como lo establece el:

\begin{abstract}
Artículo $1^{\circ}$ La Educación Superior es un proceso permanente que posibilita el desarrollo de las potencialidades del ser humano de una manera integral, se realiza con posterioridad a la educación media o secundaria y tiene por objeto el pleno desarrollo de los alumnos y su formación académica o profesional. (p. 1)
\end{abstract}

La importancia de esta ley es la figura que se le da a la educación superior antes considerada como privilegio de muy pocos en el país, para que muchos puedan acceder a ella; sumado a esto, también se estipulan los fundamentos de los estudios de posgrado como lo son las especializaciones, maestrías, doctorados y posdoctorados, mostrando una mayor preocupación e interés por que se cumpla el derecho a la educación y de esta manera puedan los estudiantes aspirar a una mejor calidad de vida.

Sin embargo, la educación en Colombia es afectada por diversas situaciones como: "la situación del país a nivel social no ha sido fácil, la violencia generada por todos los grupos al margen de la ley, (guerrilleros, paramilitares, bacrim, delincuencia común, etc...), ha causado situaciones relacionadas con el desplazamiento, las masacres colectivas, los secuestros, los combates permanentes, las negociaciones con la guerrilla (...), la paulatina polarización de la sociedad civil" (Herrera, Pinilla Díaz, \& Infante Acevedo, 2001, p. 41), las consecuencias sociales de éstos grupos armados, es privar de algunos derechos a los ciudadanos, en especial el de la educación; el desplazamiento es un fenómeno que durante décadas ha afectado a la población rural teniendo que abandonarlo todo y con ello el estudio, en el mejor de los casos, ya que también algunos niños o jóvenes de manera voluntaria y otros obligada ingresan a sus filas para combatir al Estado sin tener plena conciencia de que es lo que hacen y porque lo hacen.

Es por esto, que la sociedad en general se puede preguntar "¿qué papel ha cumplido y/o podrá cumplir la educación, dentro de un panorama al que no se le encuentran salidas claras e inmediatas?" (Herrera, Pinilla Díaz, \& Infante Acevedo, 2001, p. 41), esto ocurre porque la educación no se ha visto en la dimensión adecuada como una herramienta para ofrecer 
soluciones a los problemas sociales en un país al portas de vivir un estado de postconflicto o pos acuerdo, por medio de la cual los estudiantes tengan claro de qué manera contribuir con una convivencia en paz y sostenible en el tiempo, como también hacer respetar los Derechos Humanos, por lo que el Estado debe adoptar nuevas medidas y una política pública encaminada a fortalecer la unidad nacional y la convivencia en paz ${ }^{5}$.

\section{Competencias ciudadanas}

La educación de calidad no sólo implica la formación de un conocimiento disciplinar en los estudiantes, también incluye la formación integral como seres humanos con valores y principios que le sirvan para vivir en comunidad y de esta manera contribuya al fortalecimiento de la sociedad en general y esto debe ser a través de una formación ciudadana; por ende, para una mayor participación democrática hay que tener más demócratas y más lideres para de esta manera se espera que en una sociedad en pos conflicto se puedan resolver las diferencias siempre por la vía democrática y pacífica.

Por lo anterior, se puede entender cuál es la importancia de la aplicación en la sociedad y ante todo, la importancia de enseñar competencias ciudadanas en las aulas de clase de las IES, aplicando de esta manera el mandato constitucional que en el artículo 41 determinó que:

En todas las instituciones de educación, oficiales o privadas, serán obligatorios el estudio de la Constitución y la Instrucción Cívica. Así mismo se fomentarán prácticas democráticas para el aprendizaje de los principios y valores de la participación ciudadana. El Estado divulgará la Constitución. (Constitución Política de Colombia, p. 7)

Posteriormente con la promulgación de la Ley 107 de 1994, que reglamenta en parte el anterior artículo 41 de la C. P. C. para la educación básica y media, acerca de los temas de enseñanza de la constitución, prácticas democráticas y valores de participación ciudadana; en los artículos 2 y 3, los cuales dictan que la educación debe incluir dentro de su enseñanza la formación en competencias ciudadanas que según el Ministerio de Educación Nacional "son el conjunto de competencias y de habilidades 
cognitivas, emocionales, comunicativas e integradoras que, articuladas entre sí, hacen posible que el ciudadano actúe de manera constructiva en la sociedad democrática" (2012).

De acuerdo con lo anterior, las habilidades cognitivas, son aquellas que se desarrollan mediante el conocimiento de la Constitución y su aplicación para el conocimiento y defensa de los derechos fundamentales y fortalecer la participación democrática. Las habilidades emocionales ayudan a identificar las emociones propias y las de los demás, y tener una respuesta positiva ante diferencies situaciones sin necesidad de considerar que la violencia o la muerte son la solución para cualquier diferencia con los demás. Las habilidades comunicativas están relacionadas con las habilidades cognitivas, en el sentido que, la comunicación y las decisiones que se toman en la vida diaria, se debe haber analizado el impacto que puedan llegar a tener en nuestra vida las consecuencias de las decisiones que se toman a diario, situaciones y personas que nos rodean y en la sociedad en general, por lo que, por medio de las habilidades comunicativas debemos entender cómo es nuestro rol en la sociedad, cómo interactuamos con los demás, y si esas interacciones hacen aportes a la sociedad (Chaux, Lleras, \& Velásques, 2004).

A este conjunto de competencias y habilidades se les debe enfocar su pedagogía para lograr una convivencia en paz de toda la sociedad; ya que posiblemente si se logra llegar a un postconflicto en el país, implica que personas que han estado al margen de la ley, dejarán las armas para luego reintegrarse en la sociedad civil, siendo un proceso difícil para las dos partes; para la población colombiana y para los excombatientes de las FARC y el ELN., lo que implica que para la sociedad debe aprender a respetar el pensamiento diferente y de manera especial la vida de todas las personas por ende para los miembros de la guerrilla que se desmovilicen, y éstas para que puedan convivir deben aprender algo útil para su inserción en la sociedad que les genere los ingresos necesarios para poder vivir dignamente sin considerar siquiera volver a las armas como fuente de vida o supervivencia.

Por esta razón, lo mejor es que este proceso que se dará en el país se empiece a asimilar desde las aulas de clase y que los estudiantes comprendan que es un cambio positivo para el país agobiado por más de cien años en una constante violencia sin razón. Para ello se puede tener en cuenta que la Ley 715 de 2001, denominada como el sistema 
general de participación que busca aportar recursos económicos frescos a la educación, aunque para obtenerlos se requiere de un alto grado de formación en los funcionarios que los deben gestionar en los departamentos y municipios del país.

Con esto se establece que, el Estado reconoce la importancia de la educación y en especial la necesidad de formar ciudadanos demócratas que sirvan de sostenimiento a la democracia, por ello se puede pensar que; "... las competencias ciudadanas pretenden permitir al estudiante pasar de un modelo de imposición y repetición a procesos de construcción social, guiados por la iniciativa individual y colectiva y por la creatividad" (Mejía \& Perafán, 2006, p. 24).

Para fortalecer la educación superior el Ministerio de Educación Nacional expidió el Decreto 2566 del 10 de septiembre de $2003^{6}$, cuyo objeto se encuentra en el "Articulo 1. Condiciones mínimas de calidad.- Para obtener el registro calificado, las instituciones de educación superior deberán demostrar el cumplimiento de condiciones mínimas de calidad y de las características específicas de calidad". Con esto se está dando un punto de partida mínimo que deben cumplir las Instituciones de Educación Superior que permita tener un criterio uniforme para todas las instituciones sobre que es calidad y hacia donde debe apuntar la formación de los estudiantes.

Adicionalmente en el artículo 3 del mismo decreto determina la "justificación del programa", y da los criterios que se tienen en cuenta para la acreditación, entre ellos se encuentra el literal "a) La pertinencia del programa en el marco de un contexto globalizado, en función de las necesidades reales de formación en el país y en la región donde se va a desarrollar el programa". En donde se puede ver claramente como a los estudiantes se les debe formar para entender las necesidades reales de Colombia entre las que podemos encontrar tener un pensamiento crítico en torno a lo que queremos como país, sus problemas y soluciones; conocer el pensamiento de los demás y respetarlo, así como también entender y plantear soluciones desde su saber disciplinar a los problemas que vive el país. 
Continuando, con la situación en la que seguramente se pueda dar como consecuencia de la firma total del fin al conflicto armado, en un proceso de aceptación de la incorporación de excombatientes en la vida civil por parte de los ciudadanos y la reparación de las víctimas, entre otros. El Ministerio de Educación Nacional ha querido promover por medio del aprendizaje de las competencias ciudadanas "tres componentes o líneas de trabajo: la movilización social; el acompañamiento a las prácticas educativas y la gestión del conocimiento para el desarrollo de competencias ciudadanas" (Ministerio de Educación, 2012).

Estos tres componentes incluyen la participación tanto de estudiantes como de docentes, siendo estos últimos una parte fundamental en la formación de principios, valores y cultura de paz, mediante un estilo pedagógico que lleve al estudiante al manejo de sus emociones y al respeto de la vida y el pensamiento diferente, y para esto el Congreso expidió la Ley 1732 de 2014 que en su artículo primero consagra:

Con el fin de garantizar la creación y el fortalecimiento de una cultura de paz en Colombia, establézcase la Cátedra de la Paz en todas las instituciones educativas de preescolar, básica y media como una asignatura independiente.

Parágrafo $1^{0}$. En observancia del principio de autonomía universitaria, cada institución de educación superior desarrollará la Cátedra de la Paz, en concordancia con sus programas académicos y su modelo educativo.

Parágrafo $2^{\circ}$. La Cátedra de la Paz tendrá objetivo crear y consolidar un espacio para el aprendizaje, la reflexión y el diálogo sobre la cultura de la paz y el desarrollo sostenible que contribuya al bienestar general y el mejoramiento de la calidad de vida de la población.

Parágrafo $3^{\circ}$. La Cátedra será un espacio de reflexión y formación en torno a la convivencia con respeto, fundamentado en el artículo 20 del Pacto Internacional de Derechos Civiles y Políticos'. Teniendo en cuenta lo anterior las instituciones de educación básica, media y superior en el país además de cumplir con el fin de formar profesionales íntegros para un

7. Artículo 20 \#1. Toda propaganda en favor de la guerra estará prohibida por la ley. \#2. Toda apología del odio nacional, racial o religioso que constituya incitación a la discriminación, la hostilidad o la violencia estará prohibida por la ley. Consultado en: http://www.ohchr.org/SP/Professionallnterest/Pages/CCPR.aspx 
mejor futuro de Colombia, también están encargadas de fomentar las competencias ciudadanas en los estudiantes de una manera pacífica; en el artículo $2^{\circ}$ de la misma Ley 1732 de 2014 considera: "Para corresponder al mandato constitucional consagrado en los artículos $22^{8}$ y $41^{9}$ de la Constitución Nacional, el carácter de la Cátedra de la Paz será obligatorio".

Con esto ya se mira a un ciudadano, desde la Constitución con unas prácticas cívicas, en favor de la democracia y el desarrollo y ahora con esta Ley 1732 se fortalecen las competencias emocionales para la supremacía de una cultura que, teniendo en cuenta el presente, visibilice cual va a ser el futuro para Colombia y trabajar por él.

\section{Conclusiones}

La pedagogía acerca de las competencias ciudadanas en las universidades es un mandato constitucional y legal que debe ser reforzado, más ahora que se está trabajando para llegar a un acuerdo para el fin del conflicto, de manera que los ciudadanos al culminar sus estudios de pregrado entiendan cómo las competencias ciudadanas se convierten en una herramienta esencial para implementar la formación sobre la paz, la convivencia y el conocimiento, y respeto por los Derechos Humanos en este nuevo escenario ya que son prioritarios para que los acuerdos sean exitosos y tengan un resultado positivo para el futuro democrático, social, económico, cultural, y educativo del país.

Teniendo en cuenta la historia de Colombia y la violencia que se ha vivido por las diferentes partes que han participado del conflicto, es necesario iniciar un proceso de aceptación de excombatientes de las guerrillas en la sociedad civil, que será un proceso a largo plazo; primero, porque han afectado al país con la inconmensurable violencia que han producido; segundo porque no es fácil tratar con actores que su accionar ha impactado de manera negativa, tanto a nivel nacional como internacional y tercero, porque los excombatientes ahora pertenecerán no sólo a la vida civil, sino a la vida política del país, lo cual generará cambios en el manejo democrático e ideológico y puede causar desacuerdos en la sociedad

8. CPC Artículo 22.- La paz es un derecho y un deber de obligatorio cumplimiento.

9. CPC Articulo 41.- En todas las Instituciones de educación, oficiales o privadas, serán obligatorios el estudio de la Constitución y la instrucción cívica. Así mismo se fomentaran prácticas democráticas para el aprendizaje de los principios y valores de la participación ciudadana. El Estado divulgará la Constitución. 
colombiana que se deben discutir en espacios académicos y democráticos y de manera democrática con el poder del voto programático avalar las propuestas de los candidatos.

Es importante tener en cuenta el rol de los educadores en este proceso, entendiendo que son intermediarios del conocimiento que se imparte a los estudiantes. El nivel de tolerancia y respeto infundido por los docentes es trascendental, pues son los principales orientadores de los estudiantes y mediadores del conocimiento que ellos adquieren, por eso, es necesario que conozcan y entiendan acerca del tema del postconflicto para poder actuar de acuerdo a la nueva dinámica que en un futuro próximo se pueda dar en Colombia, cuando se concrete la firma del acuerdo que se están negociando con los grupos guerrilleros de las FARC y el ELN.

Con esto queda claro que un ciudadano no nace sino se hace. Con la sola expedición de nuevas normas no se logra dicha formación ciudadana; requiere de un serio compromiso de la familia, la sociedad y las diferentes instituciones de educación para realmente transformar a los estudiantes en verdaderos ciudadanos e impactar de esta manera con un cambio positivo en la sociedad ya que el solo hecho de modificar las normas no se transforma a las personas en líderes que planteen verdaderas soluciones a los problemas desde su área disciplinar. 


\section{Referencias bibliográficas}

Amézquita, A., Rodríguez, S., Martínez, J. C., \& González, S. (s.f.). Colombia Aprende. Disponible en: http://www.colombiaaprende.edu.co/

Asamblea Nacional Constituyente. (s.f.). Congreso de la República de Colombia. Disponible en: http://www.cna.gov.co/1741/articles-186370_constitucion_politica.pdf

Cajiao, F. (2004). La concertación de la educación en Colombia. Revista Iberoamericana de Educación. 34, 31-47.

Consejo Nacional de Acreditacion (Ley 30 de 28 de diciembre de 1992). Ley de Educacion Superior Disponible en:http://www.cna.gov.co/1741/articles-186370_ley_3092.pdf

Constitucion Politica de Colombia (1992 actualizada a 2016). Disponible en:http://wsp. presidencia.gov.co/Normativa/Documents/Constitucion-Politica-Colombia.pdf

Chaux, E., Lleras, J., \& Velásques, A. M. (2004). Competencias Ciudadanas: de los estándares al aula. Una propuesta de integración a las áreas académicas. Bogotá: Uniandes. Disponible en: http://www.colombiaaprende.edu.co/html/mediateca/1607/articles-75077_archivo.pdf

Herrera, M. C., Pinilla Díaz, A., \& Infante Acevedo, R. (2001). Conflicto Educativo y Cultura Política en Colombia. Nómadas (15), 40-49.

Ley 30 de 1992. (28 de diciembre de 1992). Ministerio de Educación. Disponible en: http:// www.mineducacion.gov.co/normatividad/1753/articles-85860_archivo_pdf.pdf

Ley 56 de 1927. (10 de Noviembre de 1927). Ministerio de Educación. Disponible en: http:// www.mineducacion.gov.co/normatividad/1753/articles-102998_archivo_pdf.pdf

Mejía, A., \& Perafán, B. (2006). Para acercarse al dragón, para amansarlo, es necesario haberlo amansado primero: Una mirada crítica a las competencias ciudadanas. Revista de Estudios Sociales (23), 23-35.

Ministerio de Educación. (25 de Junio de 2014). Mineducacion. Disponible en: http://www. mineducacion.gov.co/

Ministerio de Educación. (Decreto 2566 de 10 de septiembre de 2003). Ministerio de Educación. Disponible en: http://www.mineducacion.gov.co/1621/articles-86425_Archivo_pdf.pdf

Ministerio de Educación. (26 de Junio de 2016). Ministerio de Educación. Disponible en: http:// www.mineducacion.gov.co/1621/w3-article-

Pécaut, D. (2009). La "guerra prolongada" de las FARC. ISTOR Revista de Historia Internacional (37), 36-47.

Ruiz Silva, A., \& Chaux Torres, E. (25 de Abril de 2005). La Formación de Competencias Ciudadanas. Bogotá: Asociación Colombiana de Facultades de Educación - Ascofade. 
Tawse-Smith, D. (2008). Conflicto Armado Colombiano. Desafíos (19), 270-299.

Universidad de los Andes. (2013). Aulas en Paz. Disponible en: http://aulasenpaz. uniandes.edu.co

Vega Cantor, R. (2004). Las luchas agrarias en Colombia en la década de 1920. Cuadernos de Desarrollo Rural, 1 (52), 9-47. 